\title{
Red de aprendizaje en web para dispositivos móviles
}

\author{
Contreras Hernández, S. ${ }^{1}$, Muñoz Galicia, A. I. ${ }^{2}$ \\ ${ }^{1}$ División de Informática, Universidad Politécnica del Valle de México \\ Av. Avenida Mexiquense s/n esquina Av. Universidad Politécnica \\ Col. Villa Esmeralda, 54910 Tultitlán Estado de México. \\ 2 División de Informática, Universidad Politécnica del Valle de México \\ Av. Avenida Mexiquense s/n esquina Av. Universidad Politécnica \\ Col. Villa Esmeralda, 54910 Tultitlán Estado de México. \\ 1salvador.contreras@gmail.com,2america.i.munoz@gmail.com
}

Fecha de recepción: 30 de septiembre 2015

Fecha de aceptación: 4 de diciembre 2015

Resumen. El proyecto consiste en el desarrollo de un sistema de red de usuarios para compartir información en el ámbito educativo. El diseño de la aplicación permite la interacción con ésta a través de una computadora de escritorio o de dispositivos móviles como tabletas o teléfonos inteligentes. La información compartida es generada por alumnos a través de las actividades de aprendizaje colocadas por el profesor del curso. Las clases son compartimentos accedidos por los usuarios inscritos, ya sean alumnos, profesores o administradores del curso. El sistema está desarrollado con tecnología que permite el acceso rápido a la información contenida en la bases de datos.

Palabras clave: Red de Aprendizaje, M-Aprendizaje, Educación Móvil, Sistema Web para Dispositivos Móviles.

Summary. The project involves the development of network system users, to share information in education. The application design enables interaction with it through desktop or mobile devices such as tablets or smartphones. The shared information is generated by students through learning activities arranged by the teacher. Classes are compartments accessed by registered users, whether students, teachers or administrators of the course. The system is developed with technology that allows quick access to information contained in database.

Keywords: Learning Network, M-Learning, Mobil Education, Web System for Mobile Devices.

\section{Introducción}

El presente trabajo es un reporte del desarrollo tecnológico de la denominada Red de Aprendizaje, en su primera etapa. Consiste en un sistema orientado a la educación, sin fines de lucro, que permite compartir información generada en las clases, entre usuarios registrados.

El desarrollo del sistema móvil para la compartición de recursos y actividades de aprendizaje en el ámbito educativo, se lleva a cabo con el objetivo de hacer que la información relativa a los cursos presenciales y a distancia sea accesible a través de cualquier computadora o dispositivo móvil. De esta forma, la planeación de la clase por parte de profesor, las aportaciones de los miembros y comentarios sobre esta información, pueden ser consultados en cualquier momento y desde cualquier lugar donde se cuente con acceso a Internet. El sistema se puede utilizar en una modalidad híbrida, debido a que en un curso presencial puede servir como apoyo en el manejo de la información, tareas y contenidos.

La utilización de plataformas móviles para el apoyo a la educación se conoce como M-Learning o aprendizaje móvil en español. Sin embargo este concepto va más allá del solo desarrollo tecnológico, incluyendo aspectos pedagógicos de la educación a distancia, entre los cuales se ha demostrado (Ramos et. al., 2010) que ciertas técnicas en esta modalidad educativa son efectivas para el aprendizaje de los alumnos.

En este artículo se abarca únicamente el aspecto técnico del desarrollo del sistema, desde un punto de vista práctico. Se mencionan las tecnologías que se emplearon para el desarrollo del sistema, algunos aspectos de la programación del lado del cliente, específicamente de cómo se envía la información al servidor. Se aborda también una descripción de los componentes de la aplicación.

\section{Planteamiento del escenario}

Actualmente podemos encontrar en el mercado, tanto en software comercial como libre, plataformas y sistemas para educación a distancia, muy robustas y con muchas opciones para profesores y alumnos, algunas adaptables, y otras no tanto, a las necesidades de las instituciones o de los cursos. Lo que se pretendió atender con el proyecto de desarrollo de software aquí presentado es el acceso y compartición de información, de acuerdo a las necesidades en educación superior. El planteamiento de solución a problemas de acceso a la 
información generada en los cursos presenciales representa el motivo para el desarrollo tecnológico de la aplicación Red de Aprendizaje.

El acceso a la información, desde cualquier dispositivo, por parte de los alumnos y profesores representa un elemento crucial en la concepción de este proyecto de desarrollo tecnológico. Por parte de los alumnos, éstos tienen acceso a la información generada por todos los miembros del curso, tanto la del profesor como la de compañeros, además pueden compartir su información con otros usuarios del sistema que no están inscritos en la clase. Por su lado, el profesor puede colocar la planeación de la clase que consiste en la descripción de las actividades de aprendizaje y tiene la posibilidad de acceder en cualquier momento a las aportaciones o tareas que los alumnos hayan colocado en los apartados correspondientes. Además el profesor puede calificar en el sistema las tareas que el alumno ha enviado y en ese momento quedará registrada su calificación en la base de datos. El alumno puede consultar su calificación inmediatamente después de que el profesor la haya registrado.

El acceso a la información y al conocimiento, como se establece en Organista-Sandoval et. al.(2013), tiene que considerar como aspecto fundamental la adopción de una perspectiva que facilite la navegación permanente, lo que implica desde la conexión hasta la disponibilidad de contenidos. Las aplicaciones web para dispositivos móviles y las aplicaciones nativas tienen sus ventajas y desventajas, cada uno de estos tipos puede funcionar mejor que el otro dependiendo del problema que se intente solucionar. Por ejemplo, las aplicaciones nativas, pueden tener acceso a recursos del dispositivo como la base de datos, donde pueden guardar información de manera local, para que cuando el usuario esté desconectado pueda acceder a ella sin ningún problema. Por otra parte, las aplicaciones web para móviles tienen la ventaja de ahorrar espacio en el dispositivo móvil porque los datos los almacenan de manera externa, en un servidor remoto. La desventaja en estas aplicaciones, si es que existe, es que deben contar siempre con una conexión a Internet. Lo ideal sería combinar los dos tipos de aplicaciones, para que se pueda acceder a la información inclusive de manera desconectada.

\section{Componentes de la aplicación}

El sistema está diseñado sobre una página principal adaptable al tamaño de la pantalla del dispositivo desde el cual es accedido. Está compuesto por capas o divisiones, una de las cuales es denominada como capa principal. Esta capa es utilizada para cargar información de acuerdo a la opción del menú que se ha seleccionado. Los módulos del sistema son: Planeación, Documentos, Ligas a sitios de interés, Calificaciones y Mensajes. Se incluye un módulo adicional de Administración de la clase, para profesores únicamente. Además contiene opciones para que los usuarios se inscriban o se den de baja de una clase y para que ellos puedan crear sus propios cursos como profesores o administradores. El sistema también contiene funciones para que los usuarios inviten a otros a ser contactos de ellos, para compartir información generada en los cursos.
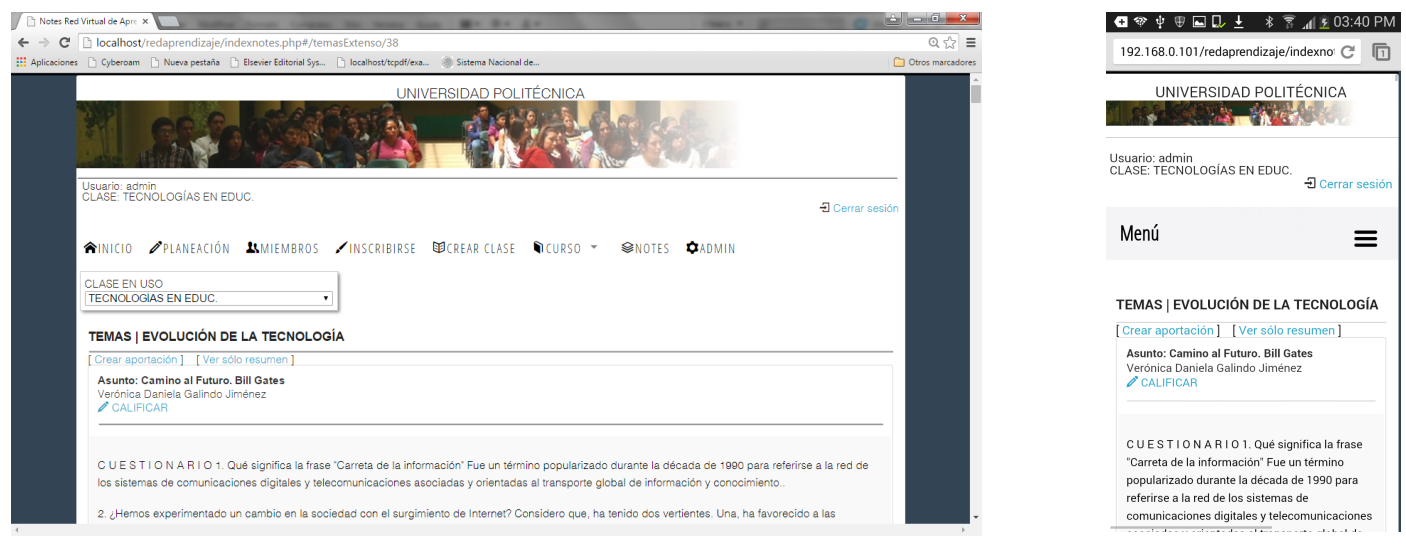

Figura 1. Interfaz de escritorio y en dispositivo móvil.

La sección de Planeación permite al profesor colocar las actividades de aprendizaje que han sido consideradas para el curso. El profesor, como creador de la clase, tiene los privilegios para editar o eliminar las actividades de aprendizaje que él ha colocado en la plataforma. La lista de actividades publicada por el profesor, es visible para todos los miembros de la clase. Los usuarios pueden colocar las tareas accediendo directamente en la actividad de aprendizaje de la lista de Planeación. Aquí se cuenta con un formulario para que el alumno pueda editar su trabajo y enviarlo a la plataforma. Los trabajos enviados quedan almacenados en la base de datos y podrán ser consultados por todos los miembros del grupo. 
Para consultar los trabajos o tareas de una actividad de aprendizaje en particular, los usuarios solo tienen que acceder a la actividad específica en la sección de Planeación. Cada tarea contiene el nombre del alumno que la envía, la fecha y la hora de envío. Además, el sistema cuenta con la opción para que el profesor califique cada una de las tareas recibidas. Los alumnos pueden consultar las calificaciones de cada tarea de manera personal, es decir, la opción de Calificaciones en el menú del alumno ejecuta una consulta para el usuario específico que está haciendo la solicitud.
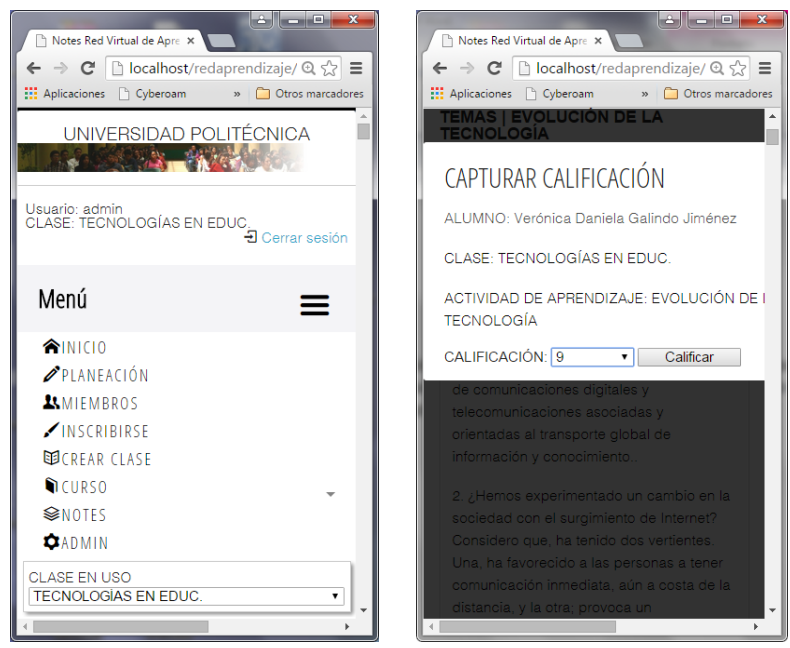

Figura 2. Menú adaptable al tamaño del dispositivo.

La sección Miembros, contiene una lista de los alumnos de la clase, que incluye nombre de usuario y foto, donde ésta tiene un vínculo al perfil del usuario. Éste contiene información del usuario como formación, escuela donde estudia, correo electrónico, foto, intereses, amigos o contactos y biografía.

Las secciones de Documentos, Ligas, Calificaciones y Mensajes se encuentran en el submenú llamado Curso, además de la sección Planeación, que aunque ya está contenida en el menú principal, se incluye aquí como acceso rápido. La sección de Ligas, contiene los enlaces a sitios de interés para la clase. La lista de sitios incluye el nombre del enlace con un vínculo al sitio de interés y una descripción. El profesor es el único que puede poner ligas en esta sección.

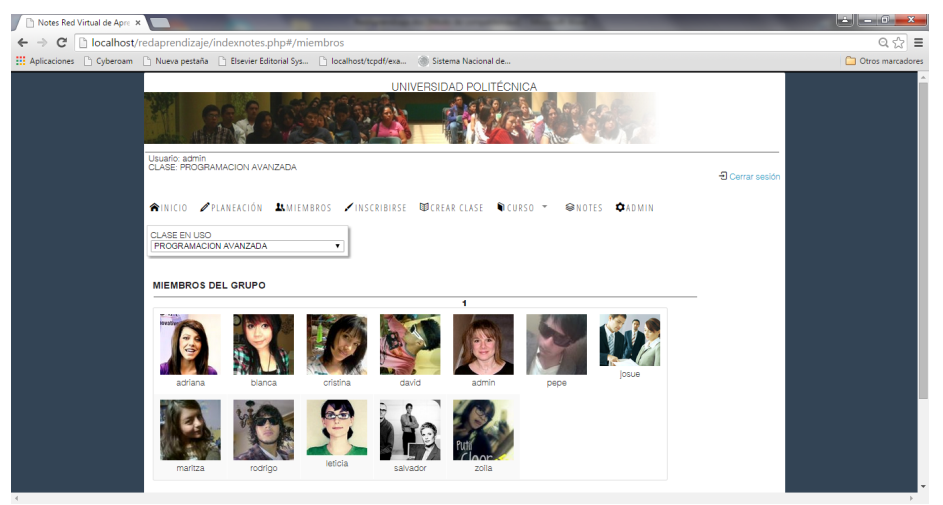

Figura 3. Miembros de clase.

Documentos es el módulo del sistema que permite a los usuarios enviar archivos a la plataforma. Los documentos enviados quedan guardados en el servidor, con una referencia en la base de datos para su localización. Los datos para el almacenamiento de los documentos son el nombre del archivo, el usuario que lo envía, la actividad de aprendizaje y la clase a la que pertenece.

En el proceso de registro de usuarios, se envían los datos al servidor, incluyendo la contraseña cifrada con el algoritmo MD5. Cuando un usuario se autentica en el sistema, la contraseña viaja cifrada y se compara con la que se tiene en la base de datos, almacenada desde que el usuario se registró. 

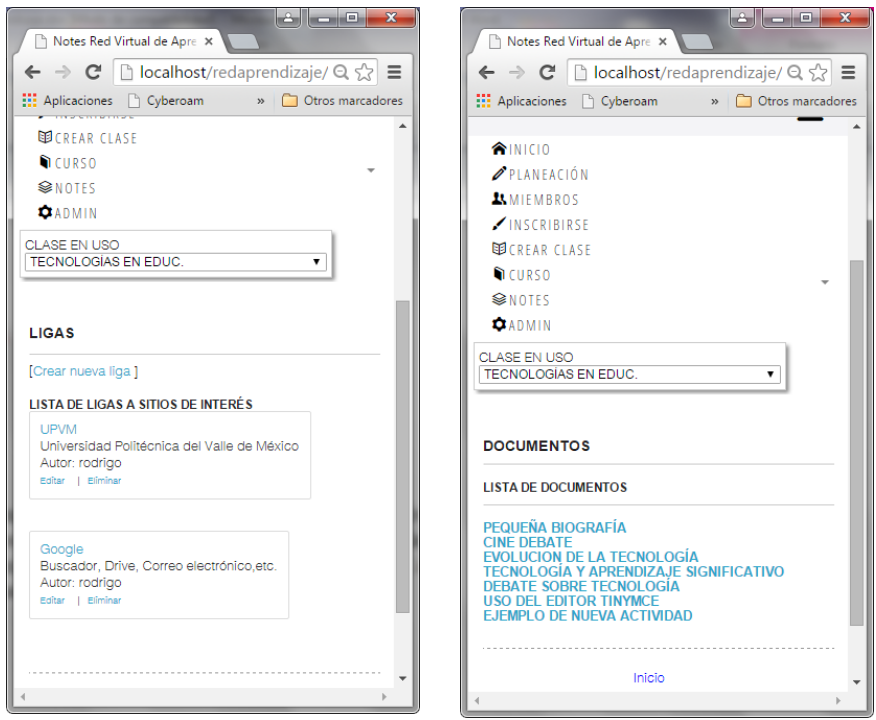

Figura 4. Vista de las secciones Ligas y Documentos.

El sistema contiene una sección para la inscripción y otra para la creación de una clase. El alumno que ya es miembro de una clase puede inscribirse a otra u otras, únicamente proporcionando la clave de la clase en el formulario de inscripción, siempre y cuando ya esté registrado como usuario del sistema. El profesor puede crear las clases que desee, además de estar inscrito como alumno en otras. El sistema distingue el rol del usuario en cada clase, ya sea profesor o alumno. El usuario que tenga varias clases, inclusive con roles distintos, tendrá una lista de selección en la página principal. Para cambiar de clase solo hay que seleccionar la correcta en esta lista. Si un usuario es alumno en una clase en uso y cambia a otra donde es profesor, no se tiene que autenticar nuevamente, el sistema contiene en su programación lo necesario para la identificación del rol en cada clase. No hay límite para el número de clases inscritas por un usuario o para el número de clases creadas.

\section{Tecnologías de la Red de Aprendizaje}

La red de aprendizaje está desarrollada con HTML 5 y JavaScript en el lado del cliente. Todas las operaciones con la base de datos están conformadas con peticiones al servidor a través de código jQuery, una librería en JavaScript para acelerar la funcionalidad a través de la web, ente otras cosas. También se usa jQuery para cargar páginas, programas e información consultada de la base de datos. La programación del lado del servidor se elaboró con el lenguaje PHP. La adaptabilidad del sistema al tamaño de la pantalla del dispositivo desde el cual es accedido, se logra con la librería Bootstrap. La metodología utilizada es un híbrido entre extreme-Programming y una adaptación considerando nuestra filosofía de trabajo en el diseño de software. Estas implementaciones híbridas son válidas y se usan más comúnmente que lo que se cree debido a que en las organizaciones se trabaja con la metodología que mejor les funciona y que es resultado de su experiencia y constantes actualizaciones, como se indica en Leiva Mundaca (2015).

Se emplean sesiones de usuario, lo cual es imprescindible, para hacer que cada persona que solicita el sitio acceda a lo que tenga permiso únicamente. Por ejemplo, las aportaciones que haga un usuario a las actividades de aprendizaje podrán ser modificadas solo por él. De la misma forma ocurre con todas las opciones donde el usuario sube información, como su perfil, comentarios a otros usuarios en la sección de las actividades de aprendizaje y documentos. Las sesiones de usuario contienen los roles a través de variables de sesión. Los profesores cuentan con opciones adicionales en el sistema para editar y eliminar comentarios, tareas y actividades de aprendizaje y aportaciones a éstas. Además, los profesores pueden colocar ligas a sitios de interés y editar información de clase por medio de la sección Administración.

El sistema está diseñado en tres capas; una para el cliente, otra para las operaciones del lado del servidor y la tercera para la base de datos. La capa del cliente contiene programas basados en jQuery para hacer las peticiones al servidor y para la interfaz de usuario. Las respuestas del servidor de MySQL se procesan a través de programas PHP que acceden a la base de datos. 

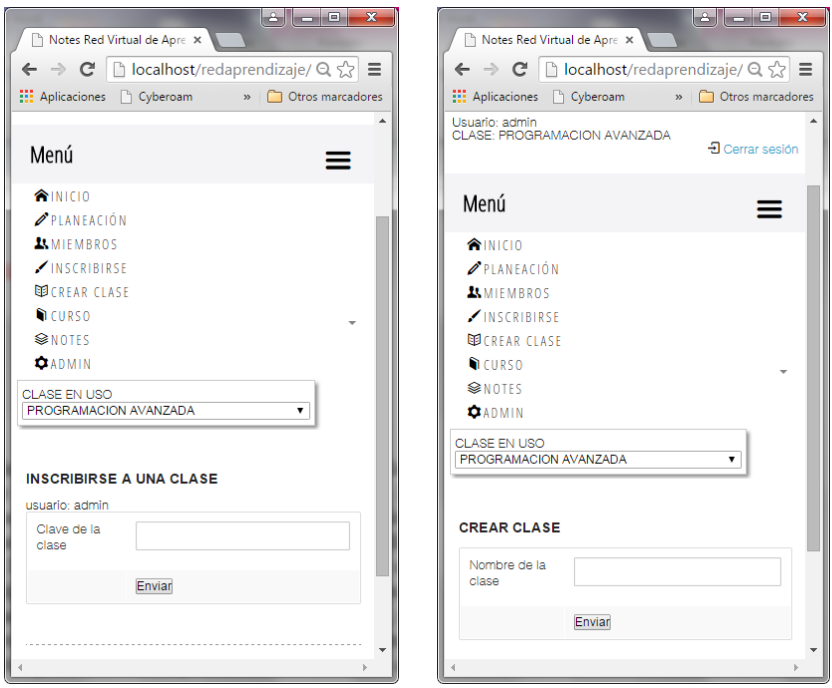

Figura 5. Módulos para inscribirse y crear una clase.

Los parámetros enviados al servidor para el trabajo con la base de datos se hacen a través de código en jQuery. Por ejemplo, para dar de alta una nueva actividad de aprendizaje, registrar un usuario, crear una aportación para un tópico, etc. También las solicitudes de consulta de información, modificación o eliminación de registros se hacen a través de jQuery. Las peticiones al servidor van acompañadas de las variables necesarias para hacer estas operaciones específicas. El cliente recibe una respuesta del servidor que indicará si la operación se llevó a cabo con éxito.

En el caso de las consultas, la petición al servidor se hace con la función getJSON de jQuery y la respuesta es enviada en formato JSON (Java Script Object Notation) por el programa que realiza la consulta en el lado del servidor. El programa cliente, recibe el resultado de la consulta con el código jQuery y lo muestra en la capa principal de la aplicación. Esta capa, al igual que todas las que componen el sitio, es adaptable al tamaño del dispositivo.

Las peticiones en el servidor son atendidas por programas PHP que realizan las operaciones en la base de datos. Cuando la solicitud es una consulta, el programa PHP almacena en un arraylist el resultado y luego lo convierte en formato JSON para devolver la respuesta al programa cliente.

Para cargar los contenidos de las diferentes secciones en la capa principal de la página de la aplicación, se utiliza una librería llamada Sammy.js, desarrollada en jQuery, que además permite el control de los botones adelante y atrás del navegador para que muestren los contenidos correctos de acuerdo a la acción elegida. Esto es necesario debido a que se trabaja con una única página en la que los contenidos se cargan en la capa principal. Cuando el usuario hace clic en una opción del menú o en cualquier hipervínculo del sitio, se ejecuta el código que permite cargar el contenido en la capa principal. El programa que se carga por defecto en la capa de contenidos es el que despliega la planeación de la clase. Este programa realiza la consulta de las actividades de aprendizaje de la clase.

Por último, la base de datos seleccionada para el sistema es MySQL por su confiablidad y la capacidad para almacenar una enorme cantidad de registros en tablas, además de ser software libre. Las versiones del software utilizado son MySQL 5.0.45, PHP 5.3, Apache Web Server 2.2.4, phpMyAdmin 2.10.2, jQuery 1.10.2 y Sammy 0.5.4. Como entorno de desarrollo se utilizó Dreamweaver CS5.

\section{Red de aprendizaje y acceso a la información}

Desde el punto de vista del acceso a la información, el sistema Red de Aprendizaje, permite a los profesores almacenar la planeación de su clase en servidores en Internet, recibir las tareas correspondientes a estas actividades a través de la misma plataforma y evaluarlas. Para los estudiantes, la plataforma les facilita el acceso a la información de la planeación, desde su celular o bien, desde cualquier dispositivo con conexión a Internet. Pueden subir sus tareas y consultarlas en el momento que deseen, así como revisar y comentar las de los demás miembros de la clase.

La red de aprendizaje se conforma con las conexiones que se establecen entre los usuarios de una clase, pero además, entre los de distintas clases. Solo basta ser miembro del sistema Red de Aprendizaje para poder acceder a la información de los usuarios que se mantengan como contactos unos de otros. La información 
almacenada de los usuarios, es la que pueden ver otros miembros de la red, es decir, tareas, aportaciones a las actividades de aprendizaje, comentarios a otros usuarios y datos del perfil. Toda esta información es configurable para determinar el acceso a ésta por parte de los contactos. Por defecto, la información puede ser accedida solo por contactos directos. Para que los contactos de segundo nivel o superior puedan tener acceso a la información de un usuario, éste deberá modificar la configuración por defecto de su cuenta en el sistema.

Por otra parte, en la tabla 1 se muestran las principales características de la Red de Aprendizaje comparadas con Nicenet, un sitio web con una orientación educativa similar. Nicenet es un sistema que permite a los usuarios la creación de clases, donde cada grupo es una pequeña comunidad virtual que comparte la información que se genera por cada miembro, desde la planeación de la clase por parte del profesor, hasta las aportaciones de los alumnos en cada uno de los temas o tópicos. Nicenet está desarrollado con una tecnología diferente para la programación del lado del cliente a la que se utilizó para la creación de la Red de Aprendizaje, ya que ésta última incorpora vistas elaboradas con Javascript a través de jQuery.

La lista de la tabla 1 muestra algunas de las ventajas del sistema sobre Nicenet, por ejemplo, la capacidad de adaptabilidad de las vistas de usuario a dispositivos móviles, la asignación de calificación a cada actividad de aprendizaje enviada por los alumnos, la consulta de calificaciones por grupo para el perfil de profesor de la clase, la reutilización de actividades de otras clases para la creación de una nueva, así como el envío de documentos y elección de contactos. Por otra parte, hay algunas características que la Red de Aprendizaje no incluye, a diferencia de Nicenet, como la opción para restringir vistas de las aportaciones de los alumnos, donde solo el profesor puede ver los envíos de los estudiantes, además la posibilidad de programar eventos por parte del profesor.

Tabla 1. Comparación de las características principales entre Nicenet y la Red de Aprendizaje

\begin{tabular}{|c|c|c|}
\hline Característica & Nicenet & Red de aprendizaje \\
\hline Adaptable a dispositivos móviles & No & $\mathrm{Si}$ \\
\hline Tópicos para subir tareas & $\mathrm{Si}$ & $\mathrm{Si}$ \\
\hline Calificaciones por actividad de aprendizaje & No & $\mathrm{Si}$ \\
\hline Calificaciones de grupo & No & $\mathrm{Si}$ \\
\hline Edición del perfil & $\mathrm{Si}$ & $\mathrm{Si}$ \\
\hline Reutilización de actividades de aprendizaje & No & $\mathrm{Si}$ \\
\hline Eliminación, modificación de clase & $\mathrm{Si}$ & $\mathrm{Si}$ \\
\hline Inscripción a una clase & $\mathrm{Si}$ & $\mathrm{Si}$ \\
\hline Ligas a sitios de interés & $\mathrm{Si}$ & $\mathrm{Si}$ \\
\hline Documentos & $\mathrm{Si}$ & $\mathrm{Si}$ \\
\hline Restricción en vistas de tópicos & $\mathrm{Si}$ & No \\
\hline Programación de eventos & $\mathrm{Si}$ & No \\
\hline Notificación de eventos & $\mathrm{Si}$ & No \\
\hline Usuario con diferentes roles en el sistema & $\mathrm{Si}$ & $\mathrm{Si}$ \\
\hline
\end{tabular}

\section{Relación con el aspecto pedagógico}

El sistema Red de Aprendizaje es un desarrollo tecnológico que ha sido concebido para el apoyo a la educación como un medio para almacenar y compartir información académica que se genera en los cursos. El proyecto no pretende, al menos por el momento, medir el aprendizaje de los alumnos por el uso de la plataforma de acuerdo a las bondades que representa, sin embargo el acercamiento de la información a los estudiantes de los cursos presenciales, por ejemplo, representa un valor agregado en su desempeño escolar. Esto se afirma, por la posibilidad de acceder a la información de los cursos las veinticuatro horas del día, los trescientos sesenta y cinco días del año, desde cualquier lugar del mundo donde haya una conexión a Internet, con dispositivos móviles o de escritorio. La información a la que puede acceder un alumno es la de sus cursos como la planeación de la clase, las actividades de aprendizaje desarrolladas y reportadas por él, así como la información de sus contactos, además de la posibilidad de estar en comunicación con los usuarios del sistema. Por su parte los profesores tienen acceso a la información de la planeación que ellos mismos han colocado, tareas reportadas por los estudiantes y comentarios o preguntas de los alumnos. 
Se puede decir que con las ventajas que brinda la plataforma se elimina la incertidumbre que implica el no contar con la descripción de la actividad, fechas de entrega y rúbrica o criterios de evaluación, ya que la información está disponible permanentemente. En cuanto al aprendizaje, no se puede afirmar que se incrementa porque aún no hay un estudio formal al respecto, pero la plataforma propicia elementos importantes para el apoyo al aprendizaje, como por ejemplo el acceso al material didáctico colocado por el profesor, además de las aportaciones a los temas y tareas enviadas por los alumnos. Por defecto, estas tareas y aportaciones son visibles por los miembros de una clase, lo que representa contar con materiales enfocados exclusivamente a los temas del curso en particular en el que estén inscritos los alumnos. Además, la incorporación de la tecnología móvil para apoyo al trabajo de estudiantes y profesores permite la convergencia de tres elementos fundamentales; espacio, tiempo y lugar, de manera que se flexibilice el proceso de aprendizaje y enseñanza (Ramírez Montoya, 2008).

Por otra parte, el profesor puede hacer retroalimentación de las tareas enviadas por sus estudiantes, al colocar comentarios asignados a cada aportación correspondiente a las actividades de aprendizaje de la planeación. El profesor deberá diseñar una estrategia adecuada en cada actividad planeada para lograr el aprendizaje de los estudiantes. La plataforma puede servir únicamente como apoyo al manejo de la información, por ejemplo, la tarea puede consistir en investigar un tema, realizar un ensayo con introducción, desarrollo y conclusiones, citas, referencias, exposición y debate por cada uno de los alumnos. La plataforma ayudará a la publicación y acceso por cualquier miembro del grupo a estos trabajos desarrollados. En el ejemplo anterior, las actividades pueden ser eminentemente presenciales, diseñadas por el profesor, en las que la medición del aprendizaje se realizará en el aula con la participación del alumno, tanto en la exposición de su trabajo como en el debate que se genere. El profesor puede asignar la calificación de la actividad en la plataforma. Las calificaciones representan datos generados en el curso, por lo que la plataforma nuevamente aquí servirá únicamente como un apoyo en el manejo de la información.

El sistema contiene la opción para evaluar las actividades contempladas en la planeación y que el alumno ha colocado en los temas o tópicos correspondientes. El alumno únicamente puede ver las calificaciones de las tareas que ha subido a la plataforma, mientras que el profesor puede ver las calificaciones detalladas por alumno, el resumen de calificaciones por grupo y un informe de pronóstico de reprobación. Este último considera el total de las actividades de aprendizaje que el profesor ha colocado en la planeación y el promedio que el estudiante lleva hasta el momento de la emisión del reporte, con lo que se hace una estimación de la calificación final. Este informe de pronóstico de reprobación, que también se podría llamar "alumnos en riesgo", considera el "ritmo" en el que al alumno ha avanzado hasta la fecha del reporte, y determina la probabilidad de que el alumno repruebe el curso en caso de que siga avanzando con el ritmo actual.

El pronóstico de reprobación es un elemento muy importante para el profesor y para las instituciones educativas, ya que se debe conocer a los alumnos que están en una situación de riesgo para tomar medidas preventivas. El peor escenario en un grupo sería que el profesor, tutor o directivo conozcan la situación delicada de los alumnos una vez que el curso ha terminado.

\section{Conclusiones y trabajos futuros}

El desarrollo del sistema, desde el punto de vista técnico, ha tenido resultados positivos hasta el momento, debido a que las acciones principales del software como se concibió desde el principio han sido implementadas. La programación de la parte del cliente ha permitido que el sistema tenga una interfaz amigable, agradable y fácil de usar. El diseño adaptable ha propiciado buenos comentarios al sistema, debido a que los usuarios que ingresan desde su dispositivo móvil, vuelven a entrar, por la comodidad y facilidad de acceso a la información en todo momento.

El proyecto contempla, en una segunda etapa, una sección para almacenar contenidos de las lecciones del curso. Este sería un nuevo módulo que incluye las operaciones de alta, consulta, modificación y eliminación de contenidos. La red de aprendizaje está actualmente en una etapa de pruebas, en la que se han registrado solo 40 usuarios para hacer la revisión de funcionamiento. Se están realizando los ajustes de los errores y oportunidades detectadas por los analistas y por los diseñadores del sistema. Se espera que la Red de Aprendizaje, sea un repositorio de actividades de aprendizaje en la primera etapa y posteriormente un repositorio de contenidos para cada curso implementado en el sistema.

La compartición de trabajos, tareas, actividades de aprendizaje y demás material generado en los cursos, se almacena en la base de datos del sistema para poder ser explotado, ahora en un entorno de red social, pero se contempla que más adelante se acceda a la información a través de un sistema de repositorio. Esto representa una solución al problema de la pérdida de la información que resulta como producto de las clases, desde ejercicios desarrollados, casos de estudio, ensayos, materiales didácticos, etc. Actualmente toda esta información no se tiene registrada o almacenada en algún sitio. 


\section{Referencias}

1. Leiva Mundaca, I., \& Villalobos Abarca, M. (2015). Método ágil híbrido para desarrollar software en dispositivos móviles. INGENIARE - Revista Chilena De Ingeniería, 23(3), 473-488.

2. Organista-Sandoval, J., Salas, L. M., \& Lavigne, G. (2013). El teléfono inteligente (smartphone) como herramienta pedagógica. Apertura: Revista De Innovación Educativa, 5(1), 1.

3. Ramírez Montoya, M. S. (2008). Dispositivos de mobile learning para ambientes virtuales: implicaciones en el diseño y la enseñanza. Apertura: Revista De Innovación Educativa, 8(9), 82-96.

4. Ramos, A. I., Herrera, J. A., \& Ramírez, M. S. (2010). Desarrollo de habilidades cognitivas con aprendizaje móvil: un estudio de casos. Comunicar, 18(34), 201-209. doi:10.3916/C34-2010-03-20

5. PR, N. (2011, November 28). Florida Technical College proporcionará tabletas iPad a estudiantes y profesores, a partir del 28 de noviembre. Hispanic PR Wire. 